\title{
Las colectas botánicas de Thomas Baillie MacDougall (1895-1973); LEGADO PARA LA BIODIVERSIDAD EN MÉXICO
}

\author{
María de Lourdes Rico-Arce ${ }^{1,3}$, Elizabeth Moreno-Gutiérrez², Alejandra Núñez-Merchand²,

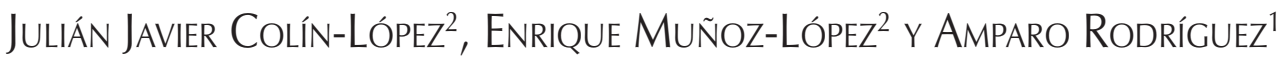 \\ ${ }^{1}$ Herbario K, Royal Botanic Gardens, Kew, Richmond, Surrey, Reino Unido \\ ${ }^{2}$ Comisión Nacional para el Conocimiento y Uso de la Biodiversidad, México, D.F., México \\ ${ }^{3}$ Autor para la correspondencia: L.Rico@kew.org
}

\begin{abstract}
Resumen: Thomas MacDougall "Don Tomás", horticultor y antropólogo de origen escocés, apasionado por la naturaleza, que de 1930 a 1973 realizó en México exploraciones científicas, principalmente en los estados de Oaxaca y Chiapas. Algunas de las plantas colectadas por él no se han vuelto a encontrar. MacDougall fue autor o coautor de géneros y de especies de plantas y animales, asimismo, al menos 21 especies de plantas se describieron en su honor. Sus colectas botánicas, incluyendo numerosos tipos, están depositadas principalmente en el Herbario del Jardín Botánico de Nueva York y en el Herbario Nacional del Instituto de Biología. La Comisión Nacional para el Conocimiento y Uso de la Biodiversidad cuenta por el momento con una base de datos de 2,601 registros, en su mayoría tomados de sus libretas de campo depositadas en Rare Book Collection del Museo de Historia Natural, de Nueva York. Debido a la alta diversidad de los lugares que visitó y a la necesidad de la conservación de su biota, se consideró importante tratar de reconstruir su itinerario botánico, ubicando y georreferenciando las localidades de sus colectas botánicas, además de realizar un análisis espacial con la vegetación primaria y el modelo digital de elevación. Como resultado, se georreferenciaron 483 sitios. Las familias mejor representadas en sus colectas en Oaxaca, Chiapas, Veracruz y Puebla, fueron: Begoniaceae, Cactaceae, Crassulaceae, Orchidaceae y Gesneriaceae. Un poco menos de 50\% del total corresponden a especies de diversas familias del bosque mesófilo de montaña en México, en el resto del porcentaje destacan principalmente aquellas del bosque de pino, pino-encino y selva baja caducifolia. La información aquí presentada es de importancia para el conocimiento, uso, y evaluación de la biodiversidad.
\end{abstract}

Palabras clave: base electrónica de datos, bosque mesófilo de montaña, colecciones históricas, conservación, georreferenciación.

Abstract: Thomas Baillie MacDougall "Don Tomas" (1895-1973) was a Scottish horticulturist, botanist and anthropologist with passion for nature. During 1930 - 1971 he explored and collected animals and plants in Mexico, mainly in the states of Oaxaca and Chiapas. To date, some of the plants he gathered have not been seen nor collected again. MacDougall was author or co-author of genera and species of plants and animals; additionally, at least 21 species were described and named after him. His botanical collections include types housed mainly in The New York Botanical Garden and the National Herbarium of the Institute of Biology in Mexico. The National Commission for the Knowledge and Use of Biodiversity has a dataset of 2,601 records, most of the information taken from his field diaries found in the Rare Book Collection of the American Museum of Natural History in New York. Due to the high diversity of the places he explored and the need to conserve species richness, we aimed to reconstruct his itinerary and georeference localities of his botanical collections using spatial analysis in search of specific places with the primary types vegetation and a Digital Elevation Model. The result was 483 georeferenced sites. The families Begoniaceae, Cactaceae, Crassulaceae, Orchidaceae and Gesneriaceae were the best represented in his collections from the states of Oaxaca, Chiapas, Veracruz and Puebla. In less degree, almost 50\% of his total collections are species found in mountane cloud forest in Mexico, the subsequent percentages were found in pine, pine-oak, and seasonally dry forests. This information herein presented is important for the use, knowledge and evaluation in the country biodiversity.

Key words: conservation, electronic database, georeferencing, historical collections, mountainous cloud forest.

T homas Baillie MacDougall (1895[6?]-1973), conocido popularmente en Tehuantepec (Oaxaca) como "Don Tomás", nació en la Isla de Bute (Escocia) el 9 de diciembre, el año de su nacimiento permanece en duda entre 1895 o
1896. Durante su juventud estudió en el Jardín Botánico de Manchester; en 1920 curso agronomía forestal en Syracuse (New York State College of Agroforestry), Nueva York y en 1930 se nacionalizó como ciudadano de los Estados Unidos 
de América. De acuerdo con los registros encontrados en sus diarios de campo, en ese año y el siguiente tuvo la oportunidad de que su sueño de explorador se realizara, al visitar los estados de Veracruz, Oaxaca y Chiapas (MacDougall, 1971). En las cuatro décadas subsecuentes, cada diciembre regresaba y colectaba en las selvas y bosques de coníferas de Oaxaca: principalmente los distritos de Juchitán, Putla, Tehuantepec y Chimalapas, fueron de los sitios favoritos de colecta, debido a la exuberancia en especies tanto animales como vegetales (MacDougall, 1971). Posteriormente se mudó de forma permanente a Oaxaca, en donde residió hasta su muerte en 1973. MacDougall se maravilló de tal manera de México y de su exótica belleza, que en 1959 decidió convertirse en ciudadano mexicano. En sus diarios de campo hacía anotaciones de una manera no convencional, como lo hacen los colectores botánicos o zoólogos de nuestros días. Debido a su interés en especies animales y de plantas ornamentales, llevaba a cabo una numeración independiente para cada taxón, clasificándolo con un prefijo; por ejemplo, A para las Cactaceae, S para colecta de semillas, $\mathrm{Ch}$ para las palmas, $\mathrm{H}$ para material de herbario, entre otros. Sus diarios de campo, depositados en la biblioteca del Museo Americano de Historia Natural de Nueva York (MAHN), registran información relativa a algunos casos en los que él no fue el colector directo, sino los habitantes de la región quienes le proporcionaban el material biológico diverso, como lagartijas, serpientes, anfibios, aráceas, orquídeas, etc. MacDougall intercambiaba estos materiales por objetos que los habitantes no conseguían fácilmente, ya que en aquellos tiempos y en esos lugares, el dinero significaba menos que los 'tesoros' apreciados, como eran las barras de chocolate ("Carlos V"), medias, velas, cigarrillos, etc. En cuanto fuera posible, MacDougall averiguaba la localidad exacta de procedencia de los organismos y lo registraba en sus notas de campo.

Sus colectas documentadas fueron entre 1936 a 1971, en los estados de Veracruz, Oaxaca y Chiapas, donde trabajó acompañado de sus tres "muchachos": Chico (Francisco Ortega Martínez), Juan Ramírez Vigueño y Cipriano Martínez, pobladores locales oaxaqueños del Istmo de Tehuantepec. A su vez, la información de la base de datos, que está basada en datos de sus libretas de campo, indica que los años de mayor productividad como colector de plantas, fueron 1963, 1964, 1967 y 1971, en los estados de Oaxaca y Chiapas.

MacDougall fue autor de notas, especies y géneros, principalmente de cactáceas y begoniáceas, pocas veces como único autor y más frecuentemente en colaboración con personajes como el Dr. Richard Alden Howard, del Arnold Arboretum en Harvard University (E.U.A.), en lo referente a plantas y, en el caso de animales, los anfibios con Edward H. Taylor (Taylor y MacDougall, 1949), reptiles con Hobart Smith y mamíferos con George G. Goodwin (Goodwin y MacDougall, 1955a, b, c, 1956a, b, 1958, 1959a, b, 1963 , 1964,1966, 1969); todos ellos, curadores asociados a los
Museos de Historia Natural en Illinois y en Nueva York; el Dr. Miguel Álvarez del Toro del Instituto de Historia Natural en Chiapas (México), fue también muy amigo y colaborador de MacDougall. En cuanto a la lista de los animales, no conocemos mucho; sin embargo, uno de los autores (LR) elabora una base electrónica en la que se están registrando las especies y citas en donde sus colecciones fueron usadas para describir los tipos taxonómicos o nuevos registros en su distribución. Asimismo, se busca la colaboración de investigadores especialistas de anfibios en la Facultad de Ciencias de la UNAM y la Universidad de Texas.

Dos años antes de su muerte se publicó The Chima Wilderness (MacDougall, 1971), donde describe la belleza de los lugares del trayecto entre el Istmo de Tehuantepec y la región de los Chimalapas. El itinerario que inició en Santa María Chimalapa y el Río Corte, continúa hasta Cerro Atravesado, Cerro Azul y Río Ostuta. En este trabajo menciona los nombres comunes y científicos, tanto de las plantas como de los animales, que él consideraba particularmente interesantes; además, describe cómo se llevó a cabo el recorrido por esas tierras en compañía de los habitantes de la región, aunado a la información de los reportes poco difundidos sobre las excursiones de Shufeldt (1872) y Williams (1852), que dan a conocer los inventarios de especies en el Istmo de Tehuantepec; recopilación extraordinaria llevada a cabo como requisito para planear las vías ferroviarias que comunicarían la región Atlántica con la Pacífica de México.

Edward Johnston Alexander, especialista y propagador de cactáceas del Jardín Botánico de Nueva York, después de jubilarse, se dedicó arduamente al estudio de las colectas de MacDougall. En virtud de los numerosos géneros y especies descritos por Alexander, MacDougall es galardonado en Albuquerque, Nuevo México, en mayo del 1967, con el Fellow Award, que otorga la Sociedad de Cactáceas y Suculentas de los Estados Unidos de América (CSSA, por sus siglas en inglés), por su labor como colector y descubridor de plantas nuevas para la ciencia (Botanical explorer of Mexico, prolific writer, describer of the genus Neodawsonia, the tree Furcreas and new taxa of Mexico).

Debido a su formación como horticultor, MacDougall conformó una colección de especímenes vivos, que fueron distribuidos a jardines botánicos e invernaderos comerciales, algunos de estos ejemplares en la actualidad se encuentran en numerosos jardines botánicos o especies comerciales de ornato; ellos corresponden a vástagos de las plantas silvestres colectadas por él en la naturaleza. En 1946 colectó un ejemplar estéril de Epiphyllum sp., mismo que al florecer en 1950, fue entonces descrito por Alexander como Cryptocereus anthonyanus Alexander, actualmente este taxón es aceptado como Selenicereus anthonyanus (Alexander) D.R.Hunt. Es importante mencionar que a la fecha no se ha encontrado un pariente cercano al mismo. Respecto a $\mathrm{Or}$ tegocactus macdougallii Alexander, descubierto en 1952 y descrito en 1961, se infería que posiblemente estaba relacio- 
nado con el género Coryphantha, pero no categóricamente y quedaba en duda su posición; recientemente los estudios de ADN confirman su parentesco con los géneros Coryphantha y Neolloydia (Butterworth y Wallace, 2004). Cabe mencionar que el nombre del género está dedicado a Francisco Ortega ("Chico"), en honor a la profunda amistad que los unía y el epíteto específico a "Don Tomás" (MacDougall).

En sus colectas se encuentran numerosos tipos, en su mayoría depositados en colecciones del extranjero, como el Jardín Botánico de Nueva York (NY), institución que en numerosas ocasiones pagaba por los materiales enviados. Posteriormente, a raíz de que conoció y estableció amistad y colaboración botánica con los doctores Faustino Miranda, Helia Bravo, Eizi Matuda, el profesor Maximino Martínez y el Lic. Hernando Sánchez-Mejorada, fue que algunos de los duplicados de sus ejemplares fueron donados al Herbario Nacional del Instituto de Biología, de la Universidad Nacional Autónoma de México (MEXU), en México. Información adicional a muchos de los quehaceres de MacDougall en el ámbito de la horticultura y su colaboración con colegas fue proporcionada por Stix $(1973,1974,1975 a, b)$.

En aquellos años las autoridades que expedían los permisos de colecta no requerían duplicados de los materiales, tampoco estaban interesadas en tenerlos, porque no había un repositorio oficial para los duplicados en México, es por ello que en lo referente a colectas zoológicas, la mayoría de estas se encuentran en el Museo Americano de Historia Natural de Nueva York (MAHN), muy pocas fueron depositadas en el museo de Chiapas (México), una vez que conoce al Dr. Miguel Álvarez del Toro, en los años 40.

MacDougall respetaba las reglas en cuanto a permisos de colecta. Copias de ellos están en los documentos depositados en la Biblioteca del MAHN; por ejemplo, el número 389, expedido el 9 de noviembre de 1950, firmado por el Dr. Roberto Llamas (Director del Instituto de Biología, UNAM). Detalles de sus viajes, procesos y trámites burocráticos con fechas, también están descritos cuidadosamente en sus diarios de campo. También colaboró con personas interesadas en antropología y textiles, y de ahí que algunas de sus colectas incluyen objetos relativos a estos temas. Fue coautor en trabajos de arqueología (Peterson y MacDougall, 1974); a pesar de que en 1962, MacDougall fue acusado de traficante ilegal de antigüedades y encarcelado en Juchitán, Oaxaca. Una vez que se probó que la acusación no era verdadera, y fue liberado, MacDougall no continuó su actividad arqueológica (Stix, 1975a).

Don Tomás entrenó a algunos de los habitantes de las regiones que visitaba, quienes en su ausencia, eran guías de otros investigadores. Tal era su fama sobre cómo encontrar especies nuevas e interesantes, que los botánicos estadounidenses iban a visitarle para que él o sus guías se unieran a las expediciones en el campo. Ejemplo de esto es W.H. Camp, que en 1936 hace colectas con Don Tomás, posteriormente Camp regresa a México para seguir colectando; sin embargo, en ausencia de MacDougall en el país, efectúa esta visita con ayuda de los guías mexicanos (Francisco Ortega Martínez y Juan Ramírez Vigueño). En 1963, Caroline Kathryn Allen, especialista en lauráceas en Nueva York, explora junto con MacDougall durante dos meses, regresando a Bronx con una copia del diario de campo de él para el Jardín Botánico de Nueva York. Con estas novedades y materiales nuevos que él obtenía, el Herbario de NY continuaba comparando algunos de sus materiales (de 1969 a 1973 fueron aproximadamente 700 especímenes, com. pers. P. Holmgren).

Los objetivos de este trabajo fueron: (1) conformar una base de datos electrónica de acceso libre, para que esta sea implementada con nombres de familias, géneros y especies de uso actual; (2) construir un itinerario de las colectas botánicas de Thomas MacDougall en México, tomando como base los datos de sus libretas de colecta; (3) documentar los tipos de vegetación en el área que MacDougall exploró y qué importancia tiene este hábitat para la biodiversidad vegetal actual; y (4) enlistar algunas de las especies que se encuentran en riesgo de sobrevivencia o extintas en el país

\section{Materiales y método}

Se capturaron los datos de ejemplares colectados por MacDougall, procedentes de las notas de campo ubicadas en MAHN. Es importante mencionar que no se revisaron sistemáticamente los herbarios donde sus materiales están depositados, pues se tendría que buscar las colectas entre los varios millones de ejemplares $(7,300,000$ en NY y 1,300,000 en MEXU). Lo que sí se incluyó fue un tarjetero de datos de aproximadamente 200 especímenes, que se compiló (19701984) por uno de los autores (LR) cuando trabajaba en el herbario del Instituto de Biología (MEXU) de la UNAM. La base formulada para este trabajo contiene 53 campos (Cuadro 1).

Se revisó cuidadosamente la ortografía y se verificaron los nombres de las familias, géneros y especies disponibles en la base, en pocos casos fue necesario recurrir a especialistas de alguna familia. También se encontraron nombres inéditos, para estos se procuró saber cuál es la identidad del material en los herbarios donde se podían encontrar sus colectas; por ejemplo: NY, TEX, US, entre otras colecciones. En otras instancias los nombres de especies que MacDouga11 asignó en las libretas de campo no fue posible ubicarlas, y el nombre de la especie se dejó como "dato original"; por ejemplo: Trigridia muetajo y Achimenes laud, se consideraron en los resultados como Trigridia sp. y Achimenes sp., ya que esas especies no existen. De manera similar se procedió con los géneros. Por otro lado se hizo una búsqueda exhaustiva en el Índice Internacional de Nombres de Especies (IPNI) de todas aquellas especies o géneros dedicados a él. Los nombres de las familias que MacDougall escribió eran de acuerdo a la clasificación de Cronquist (1981); por tanto, algunas ya no están en uso de acuerdo a la clasificación del 
Cuadro 1. Campos que incluyó la base de datos de las colectas botánicas de Thomas MacDougall.

\begin{tabular}{|c|c|c|c|c|c|}
\hline $\begin{array}{l}\text { Nombre } \\
\text { del campo }\end{array}$ & $\begin{array}{l}\text { Tipo de } \\
\text { dato }\end{array}$ & Descripción & $\begin{array}{l}\text { Nombre } \\
\text { del campo }\end{array}$ & $\begin{array}{l}\text { Tipo de } \\
\text { dato }\end{array}$ & Descripción \\
\hline SiglasColeccion & Texto & $\begin{array}{l}\text { Según catálogo electrónico } \\
\text { de la Conabio }\end{array}$ & $\begin{array}{l}\text { LongitudMinutos } \\
\text { LongitudSegundos }\end{array}$ & $\begin{array}{l}\text { Numero } \\
\text { Numero }\end{array}$ & \\
\hline NombreColeccion & Texto & $\begin{array}{l}\text { Según catálogo electrónico } \\
\text { de la Conabio }\end{array}$ & Latitutd & Numero & $\begin{array}{l}\text { Información procedente de la } \\
\text { georreferenciación 29Jun2010 }\end{array}$ \\
\hline Nombrelnstitucion & Texto & & Longitud & & Información procedente de la \\
\hline PaisColeccion & Texto & & & & georreferenciación 29Jun2010 \\
\hline EstadoColeccion & Texto & & Georreferenciacion_ & Texto & Información del campo $\mathrm{No}_{-}$ \\
\hline Valid_Col-Ins_Cat & Texto & $\begin{array}{l}\text { Validación con el catálogo } \\
\text { electrónico de la Conabio } \\
\text { (se marca con x si ha sido }\end{array}$ & Notas & & $\begin{array}{l}\text { georref, procedente de la } \\
\text { georreferenciación 29Jun2010 } \\
\text { + información CISE }\end{array}$ \\
\hline & & localizado en el catálogo) & ValidacionGeografica & Texto & Validación con la carta con \\
\hline NumeroDeColecta & Texto & $\begin{array}{l}\text { Información original del } \\
\text { campo org-col }\end{array}$ & Altitud & Texto & $\begin{array}{l}\text { buffer de } 2 \text { km (Conabio, 1998) } \\
\text { Información original }\end{array}$ \\
\hline $\begin{array}{l}\text { Colector_Apellido } \\
\text { Paterno }\end{array}$ & Texto & Información depurada & Corr_altitud & Texto & $\begin{array}{l}\text { Información procedente de la } \\
\text { georreferenciación 29Jun2010 }\end{array}$ \\
\hline $\begin{array}{l}\text { Colector_Apellido } \\
\text { Materno }\end{array}$ & Texto & Información depurada & AltitudEjemplar_m & Numero & $\begin{array}{l}\text { Unidades en metros. Para } \\
\text { México no mayor a 5,747 m }\end{array}$ \\
\hline Colector_Nombre & Texto & Información depurada & & & que es la altitud del Pico de \\
\hline DiaColecta & Numérico & $\begin{array}{l}\text { Información original del } \\
\text { campo dia }\end{array}$ & & & $\begin{array}{l}\text { Orizaba y para otros países } \\
\text { deberá ser menor a } 8,840 \mathrm{~m}\end{array}$ \\
\hline MesColecta & Numérico & $\begin{array}{l}\text { Información original del } \\
\text { campo mes }\end{array}$ & & & $\begin{array}{l}\text { que corresponde al Monte } \\
\text { Everest. Un ft }=0.3048 \mathrm{~m}\end{array}$ \\
\hline AnioColecta & Numérico & $\begin{array}{l}\text { Información original del } \\
\text { campo año }\end{array}$ & $\begin{array}{l}\text { Altitud_Final } \\
\text { Ejemplar_m }\end{array}$ & Numero & \\
\hline obsv & Texto & Información original & TIP_VEG & Texto & Dirección General de \\
\hline Reino & Texto & & & & Geografía - Instituto Nacional \\
\hline Division & Texto & & & & de Estadística, Geografía e \\
\hline Clase & Texto & & & & Informática (INEGI). 2003. \\
\hline Orden & Texto & & & & Vegetación Primaria. Escala \\
\hline Familia & Texto & Información actualizada APG III & & & 1:1000000. Instituto Nacional \\
\hline Genero & Texto & & & & de Estadística, Geografía e \\
\hline Especie & Texto & & & & Informática - INEGI. \\
\hline Familia_original & Texto & Información original & & & Aguascalientes, Ags., México \\
\hline Genero_original & Texto & Información original & Analista & Texto & Persona que asignó las \\
\hline Especie_original & Texto & Información original & & & coordenadas \\
\hline Pais & Texto & Nombre del país de colecta & Fecha & Fecha/ & Fecha de georreferenciación \\
\hline Estado & Texto & $\begin{array}{l}\text { Información procedente de la } \\
\text { georreferenciación 29Jun2010 }\end{array}$ & Incertidumbre & $\begin{array}{l}\text { tiempo } \\
\text { Numero }\end{array}$ & $\begin{array}{l}\text { de la localidad } \\
\text { Incertidumbre de la }\end{array}$ \\
\hline ClaveEstado & Texto & Según INEGI & & & coordenada asignada calculada \\
\hline Distrito & Texto & $\begin{array}{l}\text { Información procedente de la } \\
\text { georreferenciación 29Jun2010 }\end{array}$ & Observaciones & Memo & $\begin{array}{l}\text { en kilómetros } \\
\text { Descripción general de los }\end{array}$ \\
\hline Municipio & Texto & $\begin{array}{l}\text { Información procedente de la } \\
\text { georreferenciación 29Jun2010 }\end{array}$ & & & $\begin{array}{l}\text { criterios utilizados: caminos, } \\
\text { entronques, rasgos topográficos, }\end{array}$ \\
\hline ClaveMunicipio & Numero & Según INEGI & & & localidades homónimas, etc. \\
\hline Localidad & Texto & $\begin{array}{l}\text { Información procedente de la } \\
\text { georreferenciación 29Jun2010 } \\
\text { del campo original pueblo }\end{array}$ & Id_Formulario & Numero & $\begin{array}{l}\text { Identificador que relaciona a la } \\
\text { localidad georreferenciada con } \\
\text { la imagen }\end{array}$ \\
\hline LatitudGrados & Numero & & Mapa & Hypelink & Imagen que muestra la \\
\hline LatitudMinutos & Numero & & & & ubicación de la(s) \\
\hline LatitudSegundos & Numero & & & & coordenada(s) sitio \\
\hline LongitudGrados & Numero & & Restricciones & Text & \\
\hline
\end{tabular}




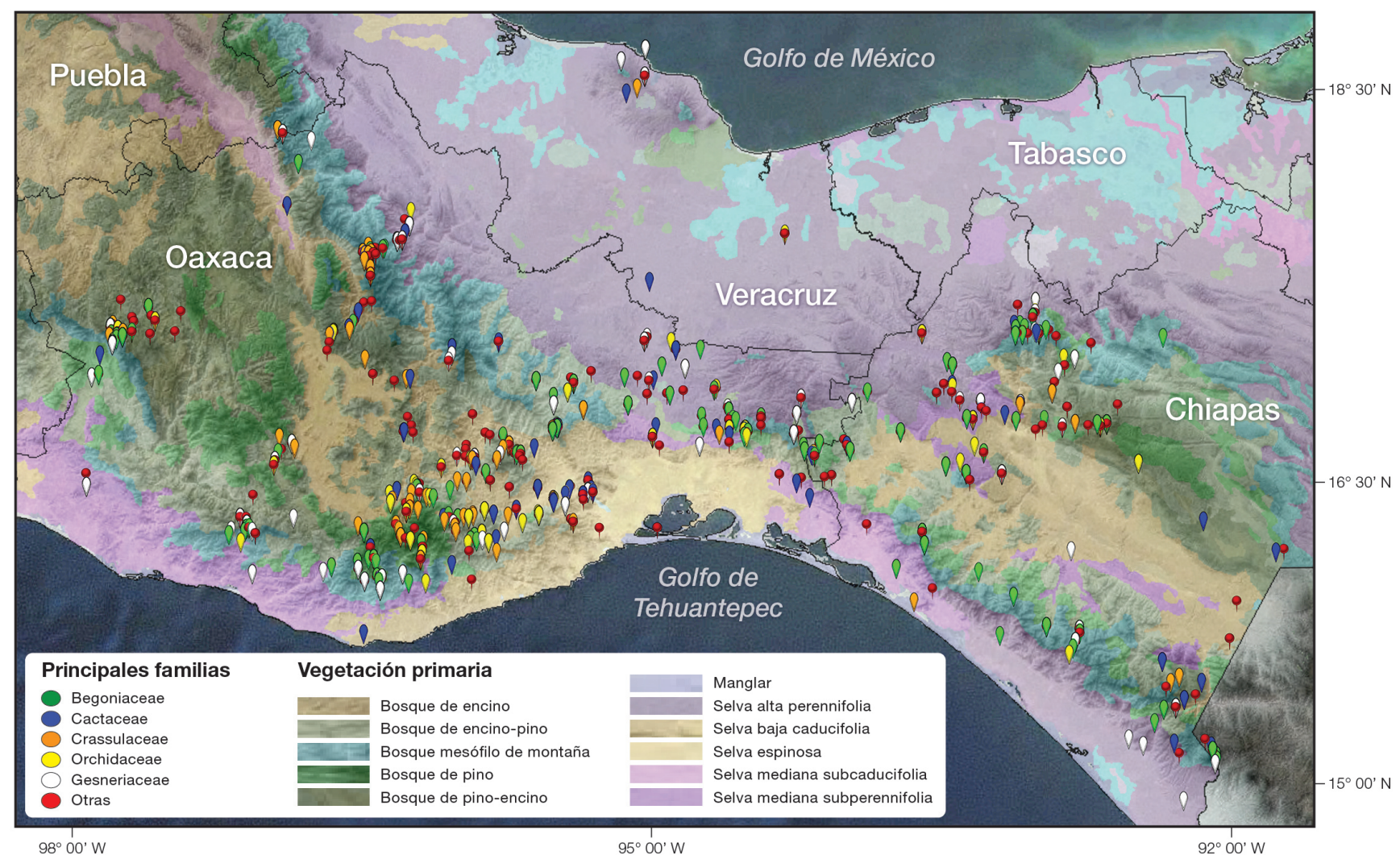

Figura 1. Distribución de sitios de colecta por familia y tipo de vegetación primaria.

Angiosperms Phylogetics Group III (APG III); por lo que fue necesario tener una tabla de equivalencias en la base formulada; por ejemplo, lo registrado como Asclepiadaceae es considerada ahora subfamilia Asclepiadoideae en las Apocynaceae. En lo referente a las especies que se encuentran en riesgo se consultó la lista de Norma Oficial Mexicana NOM-059-SEMARNAT-2010 (SEMARNAT, 2010).

Con respecto a las localidades de colecta, se georreferenciaron aplicando el método punto-radio, el cual consiste en asignar una coordenada geográfica y un valor de incertidumbre asociado a cada ubicación (Wieczorek et al., 2004). En este método, el cálculo de la incertidumbre considera variables geográficas que involucran la precisión, tanto de la cartografía usada para calcular coordenadas geográficas como de la información referida en la localidad. Adicionalmente, se siguieron los criterios para georreferenciar según el tipo de localidad, documentados en el Manual de Georreferenciación (CONABIO, 2008). La mayoría de las localidades de MacDougall hacen alusión a rasgos geográficos como poblados, ríos y cerros, algunos de los cuales incluyen el dato de altitud. Este último se consideró en la georreferenciación de dos maneras: una fue ubicando sobre la cota de nivel y otra ampliando la incertidumbre a la altitud indicada.

Por otra parte, cuando los topónimos especificados en la localidad fueron homónimos, se recurrió a la consulta de fechas y números de colecta, con el fin de elegir la localidad homónima que por ubicación se apegara más a otras localidades registradas para el mismo día o días cercanos. Algunas localidades no pudieron georreferenciarse, principalmente por ambigüedad, falta de precisión en la localidad, no se especifica municipio o porque los topónimos mencionados corresponden a nombres locales que no están registrados en la cartografía. El porcentaje de ellas se da en los resultados. Se consideraron todos los registros de la base, tuvieran o no asignado taxón, ya que estos fueron útiles debido a que sí tienen localidad asignada.

Se intentó ordenar y agrupar los registros de la base de datos por fecha y número de colecta, con el fin de identificar itinerarios, pero solo se obtuvieron fragmentos de estos. También, a partir de la ubicación de los sitios de colecta, se realizó un análisis espacial por medio de la intersección con el mapa de vegetación primaria (INEGI, 2003) y el Modelo Digital de Elevación Global (ASTER, 2009), lo cual permitió asociar a cada ubicación a una altitud y tipo de vegetación para, posteriormente, relacionar en conjunto la distribución de las localidades georreferenciadas según los tipos de vegetación y rangos de altitud.

\section{Resultados}

La base de datos de MacDougall quedó conformada, en total, por 53 campos y 2,601 registros. De este total, 199 regis- 
Cuadro 2. Número de registros/especímenes por familia botánica de acuerdo a la clasificación de Cronquist (1981) y la del APG III. Familias separadas por "l", son los nombres alternativos de acuerdo a su nombre conservado por el Código Internacional de Nomenclatura para algas, hongos y plantas.

\begin{tabular}{|c|c|c|c|c|c|}
\hline $\begin{array}{l}\text { Familia registrada } \\
\text { originalmente }\end{array}$ & $\begin{array}{l}\text { Familia de acuerdo } \\
\text { al APG III }\end{array}$ & $\begin{array}{r}\text { Número } \\
\text { de registros/ } \\
\text { ejemplares }\end{array}$ & $\begin{array}{l}\text { Familia registrada } \\
\text { originalmente }\end{array}$ & $\begin{array}{l}\text { Familia de acuerdo } \\
\text { al APG III }\end{array}$ & $\begin{array}{r}\text { Número } \\
\text { de registros/ } \\
\text { ejemplares }\end{array}$ \\
\hline Acanthaceae & Acanthaceae & 34 & Liliaceae & Liliaceae & 5 \\
\hline Agavaceae & Asparagaceae & 9 & Loasaceae & Loasaceae & 3 \\
\hline Amaranthaceae & Amaranthaceae & 1 & Loganiaceae & Loganiaceae & 2 \\
\hline Amarilidaceae & Amaryllidaceae & 1 & Loranthaceae & Loranthaceae & 1 \\
\hline Anacardiaceae & Anacardiaceae & 1 & Lythraceae & Lythraceae & 5 \\
\hline Annonaceae & Annonaceae & 2 & Magnoliaceae & Magnoliaceae & 14 \\
\hline Apocynaceae & Apocynaceae & 2 & Malpighiaceae & Malpighiaceae & 5 \\
\hline Aquifoliaceae & Aquifoliaceae & 4 & Malvaceae & Malvaceae & 40 \\
\hline Araceae & Araceae & 89 & Marattiaceae & Marattiaceae & 2 \\
\hline Araliaceae & Araliaceae & 10 & Melastomataceae & Melastomataceae & 24 \\
\hline Aristolochiaceae & Aristolochiaceae & 1 & Meliaceae & Meliaceae & 1 \\
\hline Asclepiadaceae & Apocynaceae & 5 & Moraceae & Moraceae & 4 \\
\hline Basellaceae & Basellaceae & 1 & Musaceae & Musaceae & 4 \\
\hline Begoniaceae & Begoniaceae & 391 & Myricaceae & Myricaceae & 1 \\
\hline Berberidaceae & Berberidaceae & 2 & Myrtaceae & Myrtaceae & 11 \\
\hline Bignoniaceae & Bignoniaceae & 20 & Nyctaginaceae & Nyctaginaceae & 1 \\
\hline Bixaceae & Bixaceae & 1 & Olacaceae & Olacaceae & 1 \\
\hline Bombacaceae & Malvaceae & 1 & Oleaceae & Oleaceae & 1 \\
\hline Boraginaceae & Boraginaceae & 3 & Onagraceae & Onagraceae & 22 \\
\hline Bromeliaceae & Bromeliaceae & 39 & Orchidaceae & Orchidaceae & 176 \\
\hline Buddlejaceae & Buddlejaceae & 4 & Oxalidaceae & Oxalidaceae & 5 \\
\hline Burseraceae & Burseraceae & 20 & Palmae/Arecaceae & Palmae/Arecaceae & 55 \\
\hline Cactaceae & Cactaceae & 263 & Papaveraceae & Papaveraceae & 1 \\
\hline Campanulaceae & Campanulaceae & 10 & Passifloraceae & Passifloraceae & 14 \\
\hline Cannacea & Cannaceae & 2 & Piperaceae & Piperaceae & 2 \\
\hline Capparaceae & Capparaceae & 4 & Podocarpaceae & Podocarpaceae & 3 \\
\hline Caprifoliaceae & Caprifoliaceae & 1 & Polemoniaceae & Polemoniaceae & 2 \\
\hline Caryophyllaceae & Caryophyllaceae & 1 & Polygalaceae & Polygalaceae & 2 \\
\hline Celastraceae & Celastraceae & 3 & Polygonaceae & Polygonaceae & 1 \\
\hline Clethraceae & Clethraceae & 2 & Portulacaceae & Portulacaceae & 4 \\
\hline Cobaeaceae & Polemoniaceae & 10 & Rafflesiaceae & Rafflesiaceae & 1 \\
\hline Combretaceae & Combretaceae & 5 & Ranunculaceae & Ranunculaceae & 9 \\
\hline Commelinaceae & Commelinaceae & 17 & Rhamnaceae & Rhamnaceae & 3 \\
\hline Compositae/Asteraceae & Compositae/Asteraceae & 105 & Rosaceae & Rosaceae & 13 \\
\hline Convallariaceae & Asparagaceae & 1 & Rubiaceae & Rubiaceae & 36 \\
\hline Convolvulaceae & Convolvulaceae & 27 & Rutaceae & Rutaceae & 1 \\
\hline Crassulaceae & Crassulaceae & 185 & Scrophulariaceae & Scrophulariaceae & 30 \\
\hline Cucurbitaceae & Cucurbitaceae & 1 & Simaroubaceae & Simaroubaceae & 2 \\
\hline Cyclanthaceae & Cyclanthaceae & 5 & Solanaceae & Solanaceae & 42 \\
\hline Ericaceae & Ericaceae & 19 & Sterculiaceae & Malvaceae & 8 \\
\hline Euphorbiaceae & Euphorbiaceae & 39 & Styracaceae & Styracaceae & 3 \\
\hline Fagaceae & Fagaceae & 77 & Symplocaceae & Symplocaceae & 5 \\
\hline Gentianaceae & Gentianaceae & 2 & Theaceae & Theaceae & 3 \\
\hline Geraniaceae & Geraniaceae & 2 & Theophrastaceae & Primulaceae & 1 \\
\hline Gesneriaceae & Gesneriaceae & 221 & Tiliaceae & Malvaceae & 1 \\
\hline Gramineae/Poaceae & Gramineae/Poaceae & 2 & Tropaeolaceae & Tropaeolaceae & 2 \\
\hline Gunneraceae & Gunneraceae & 2 & Turneraceae & Passifloraceae & 2 \\
\hline Guttiferae & Clusiaceae & 3 & Ulmaceae & Ulmaceae & 1 \\
\hline Hippocastanaceae & Sapindaceae & 1 & Umbelliferae/Apiaceae & Apiaceae/Apiaceae & 4 \\
\hline Hydrangeaceae & Hydrangeaceae & 1 & Urticaceae & Urticaceae & 9 \\
\hline Hydrophyllaceae & Hydrophyllaceae & 2 & Verbenaceae & Verbenaceae & 7 \\
\hline Iridaceae & Iridaceae & 8 & Violaceae & Violaceae & 1 \\
\hline Labiatae & Lamiaceae & 21 & Viscaceae & Santalaceae & 1 \\
\hline Lauraceae & Lauraceae & 17 & Vochysiaceae & Vochysiaceae & 1 \\
\hline Leguminosae/Fabaceae & Leguminosae/Fabaceae & 93 & Winteraceae & Winteraceae & 2 \\
\hline Lentibulariaceae & Lentibulariaceae & 5 & Zamiaceae & Zamiaceae & 1 \\
\hline Liliaceae & Liliaceae & 1 & Sin datos de familia & $\mathrm{N} / \mathrm{A}$ & 199 \\
\hline Liliaceae & Iridaceae & 2 & & & \\
\hline
\end{tabular}


tros no tienen identificación a familia, género o especie. En el campo especie hay nombres que no pudieron confirmarse por medio de catálogos de plantas, floras, monografías o revisiones, consecuentemente solo se validaron 730 nombres de especies. En cuanto a géneros, se logró corroborar 373 nombres. Referente a las familias, de acuerdo a la clasificación del APG III, son 106. El número de registros/especímenes por familia en ambos casos se localiza en el cuadro 2. Las familias más abundantes son Begoniaceae (391), Cactaceae (263) y Gesneriaceae (221). Por otro lado, se encontró que al menos 21 taxa de plantas han sido dedicados a él.

Del número total $(2,601)$ de registros de la base, únicamente 1,996 pudieron ser georreferenciados. El 23\% (605) de los registros no fue posible georreferenciarlo por imprecisión o por falta de información de la localidad. Frecuentemente, MacDougall visitaba el mismo lugar, por ello los registros con coordenadas geográficas fue de sólo 483 sitios. En la figura 1 se muestra la distribución de sitios de colecta de cinco familias de plantas, que son las que tienen mayor número de registros georreferenciados y los tipos de vegetación primaria donde se desarrollan, tomando como base el mapa "México: Imagen desde el espacio" (CONABIO, 2003).

Es oportuno hacer notar que muchas colectas solo consistieron en semillas o materiales frescos para jardines botánicos, por lo que no existe un ejemplar de herbario a pesar de estar registrado en la base de datos desarrollada para esta contribución. Debido al enfoque profesional como horticultor, MacDougall tenía una forma especial de numerar sus colecciones (vivas, herborizadas, semillas, etc.), con no- menclatura peculiar, ya que para cada una seguía diferentes secuencias numéricas, lo que no permite establecer una ruta cronológica de colecta completa, solo se identificaron fragmentos de sus itinerarios. Por otra parte, son pocos los registros que indican días consecutivos en las exploraciones, prevalece la separación de más de dos días en los registros consecutivos que conforman la base de datos. Esto puede deberse a la particularidad de MacDougall en su trabajo como recolector o a que existe material aún no documentado o digitalizado. Un ejemplo de sus itinerarios se muestra en la figura 2 y el cuadro 3 .

Con el análisis espacial se identificaron 11 tipos de vegetación primaria para los estados de Chiapas, Oaxaca, Puebla y Veracruz (Cuadro 4). La comunicad vegetal que MacDougall y colaboradores exploraron con mayor frecuencia en estos cuatro estados de la República Mexicana corresponde al bosque mesófilo de montaña (108 sitios), seguido del bosque de pino-encino (103 sitios) y de la selva baja caducifolia (77 sitios; Cuadro 5). No hay diferencia para el número de los sitios de colecta entre los dos primeros tipos de vegetación; sin embargo, sí para el número de especímenes, 610 y 387 respectivamente. También se identificó que el gradiente altitudinal va desde el nivel del mar hasta 3,328 m.

\section{Discusión}

Respecto a la nomenclatura de los ejemplares que se tiene en la base de datos, en la que se incluyen géneros y especies, una buena parte de estos registros necesitan actualizar-

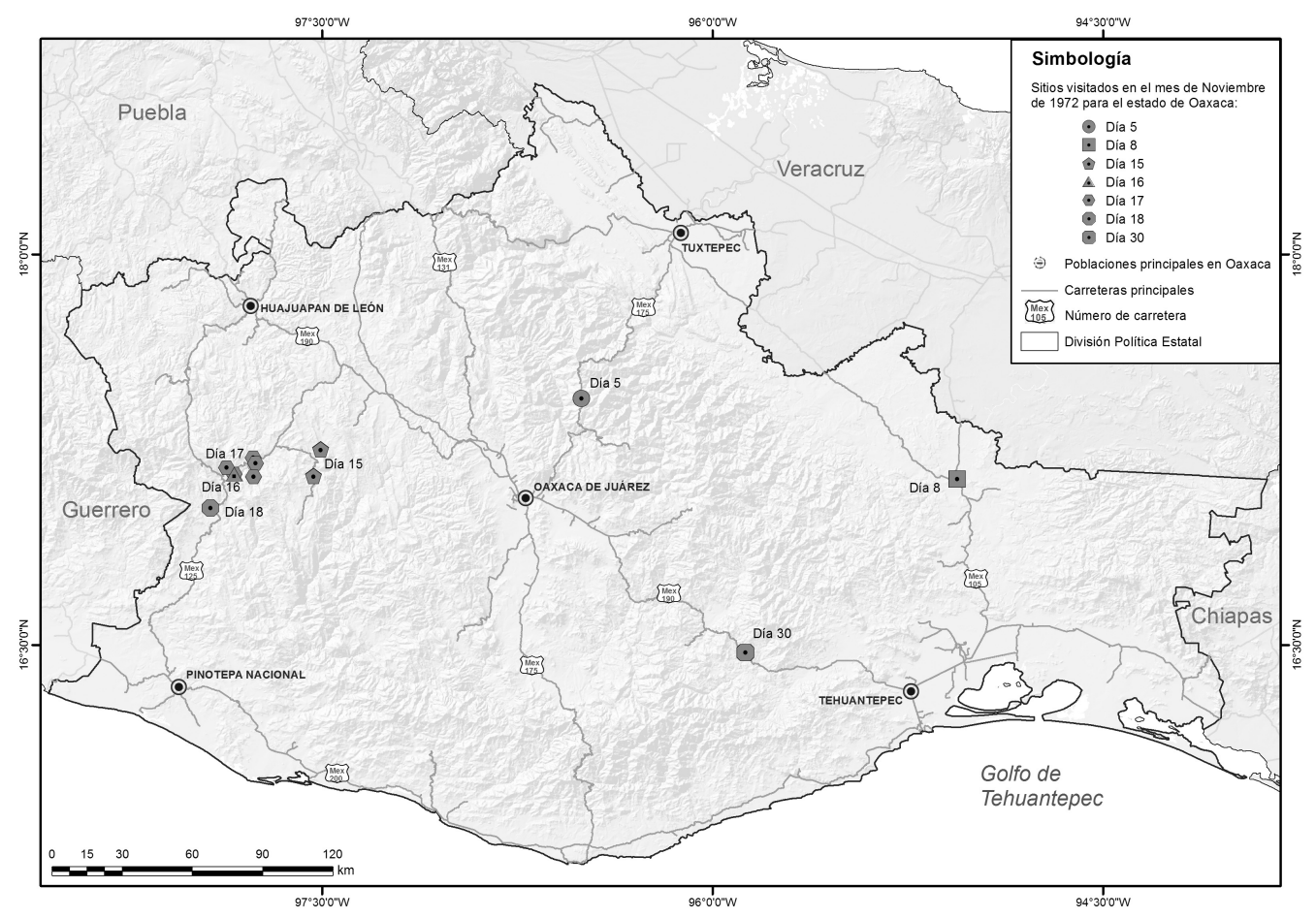

Figura 2. Representación de uno de los itinerarios de MacDougall en el estado de Oaxaca. 
Cuadro 3. Secuencia del mes de Noviembre de 1972 para el estado de Oaxaca. Las letras de la segunda columna corresponden a la clave que MacDougall usaba para Begoniaceae (B), semilla (s) y espécimen de herbario $(\mathrm{H})$, inicialmente él usaba un prefijo, finalmente solo uso número. Las localidades con asterisco al final $\left({ }^{*}\right)$, no pudieron georreferenciarse.

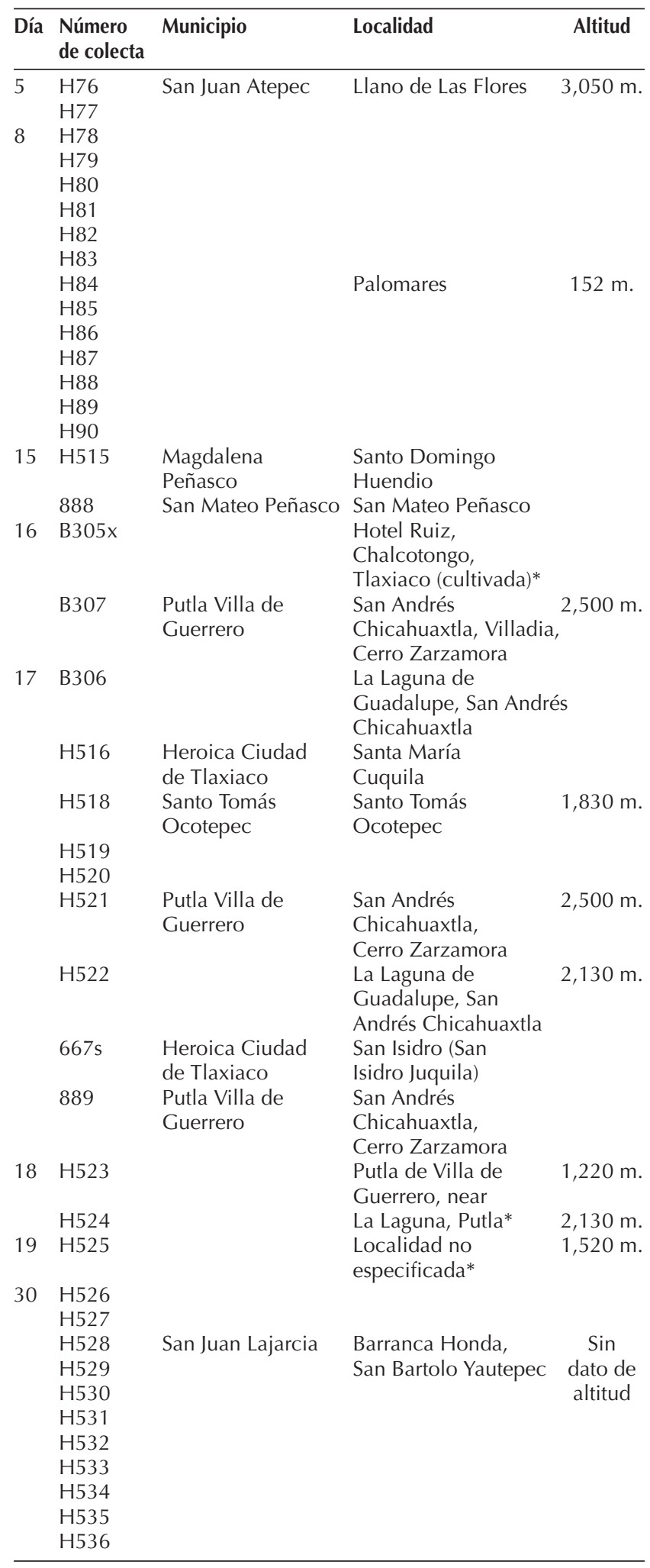

Cuadro 4. Tipos de vegetación primaria de acuerdo a los registros/ ejemplares y sitios de colecta de 1994, 607 ejemplares del total de la base no fue posible ubicarlos en un tipo particular de vegetación.

\begin{tabular}{lrr}
\hline Vegetación primaria * & Ejemplares & $\begin{array}{r}\text { Sitios de } \\
\text { colecta }\end{array}$ \\
\hline Bosque mesófilo de montaña & 610 & 108 \\
Bosque de pino-encino & 387 & 103 \\
Selva baja caducifolia & 335 & 77 \\
Selva alta perennifolia & 225 & 55 \\
Bosque de pino & 178 & 56 \\
Bosque de encino-pino & 76 & 13 \\
Bosque de encino & 73 & 28 \\
Selva mediana subcaducifolia & 50 & 22 \\
Selva mediana subperennifolia & 47 & 10 \\
Manglar & 8 & 3 \\
Selva baja espinosa & 5 & 4 \\
\hline
\end{tabular}

se taxonómicamente con la colaboración de especialistas; ya que desde que fueron registrados en los diarios de campo o en los materiales de herbario, muchos han sido reidentificados o cambiaron de ubicación taxonómica. Por ejemplo: Omiltemia scoti J.H.Kirkbr. (MacDougall H.399, tipo depositado en NY) fue descrita en honor de MacDougall (Kirkbride, 1984); con el epíteto genitivo de scotus, persona de ascendencia escocesa. Tal especie, años más tarde, fue ubicada como Deppea scoti (J.H.Kirkbr.) Lorence (Lorence y Dwyer, 1988) y actualmente se acepta con el binomio de Bellizinca scoti (J.H.Kirkbr.) Borhidi (Borhidi et al., 2004). Este ejemplo evidencia que la depuración de los nombres no puede ser sólo automática; es decir, buscando electrónicamente todos los epítetos "macdougallii".

Sobresalen por su abundancia los ejemplares de Begoniaceae (391) y Cactaceae (263). Los taxa de ambas familias fueron solicitadas por Rudolf Zeisenhenne (invernaderos particulares en Santa Bárbara, California) y E.J. Alexander (especialista y propagador de plantas en el Jardín del Bronx, N.Y., E.U.A.), en los primeros viajes de MacDougall, según los datos de sus diarios de campo.

Se conoce que un gran porcentaje de especies nuevas se encuentran o están por descubrirse en los acervos de herbarios (Bebber et al., 2010). Tomando de ejemplo algunos especímenes colectados por MacDougall se sabe de un espécimen anotado por John Fay en 1977, en NY y TEX como tipo de Oteiza macdougallii, sp. ined., que aun continua inédito; Fay (1978) publica el tratamiento de Perymenium, género emparentado con Oteiza, sin mencionar la colección de MacDougall aludida, de tal manera que la especie nunca fue formalizada (o descrita oficialmente). Por su parte Robinson (1979) opina que algunos elementos de Oteiza pertenecían a Schistocarpha; posteriormente, cuando él revisa las especies, retracta su opinión en cuanto a que Oteiza = Schistocarpha, aceptando que es un error y que Oteiza merece ser un género aceptado y diferente a Schistocarpha. Panero et al. (1993) describen dos nuevas especies de Otei- 
Cuadro 5. Intervalo altitudinal por vegetación primaria para cada estado con base en los sitios de colecta.

\begin{tabular}{|c|c|c|c|c|}
\hline Estado & Vegetación primaria & $\begin{array}{l}\text { Intervalo altitudinal } \\
\quad(\mathrm{m} \text { s.n.m.) }\end{array}$ & Ejemplares & $\begin{array}{r}\text { Sitios de } \\
\text { colecta }\end{array}$ \\
\hline \multirow[t]{10}{*}{ Chiapas } & Bosque de encino & $447-1,633$ & 10 & 5 \\
\hline & Bosque de encino-pino & $1,770-2,716$ & 52 & 7 \\
\hline & Bosque de pino & $988-2,205$ & 13 & 7 \\
\hline & Bosque de pino-encino & $733-2,581$ & 61 & 18 \\
\hline & Bosque mesófilo de montaña & $482-3,034$ & 179 & 34 \\
\hline & Manglar & 5 & 1 & 1 \\
\hline & Selva alta perennifolia & $17-1,669$ & 54 & 21 \\
\hline & Selva baja caducifolia & $411-1,134$ & 76 & 16 \\
\hline & Selva mediana subcaducifolia & $20-1,003$ & 9 & 6 \\
\hline & Selva mediana subperennifolia & $729-1,171$ & 41 & 6 \\
\hline \multirow[t]{11}{*}{ Oaxaca } & Bosque de encino & $421-2,244$ & 63 & 23 \\
\hline & Bosque de encino-pino & $893-2,380$ & 24 & 6 \\
\hline & Bosque de pino & $378-3,260$ & 165 & 49 \\
\hline & Bosque de pino-encino & $372-3,328$ & 326 & 85 \\
\hline & Bosque mesófilo de montaña & $440-3,197$ & 424 & 72 \\
\hline & Manglar & $1-4$ & 7 & 2 \\
\hline & Selva alta perennifolia & $39-1,434$ & 138 & 29 \\
\hline & Selva baja caducifolia & $15-2,261$ & 259 & 61 \\
\hline & Selva baja espinosa & $16-1,561$ & 5 & 4 \\
\hline & Selva mediana subcaducifolia & $54-592$ & 41 & 16 \\
\hline & Selva mediana subperennifolia & $477-1,222$ & 6 & 4 \\
\hline Puebla & Bosque mesófilo de montaña & 2,153 & 3 & 1 \\
\hline \multirow[t]{2}{*}{ Veracruz } & Bosque mesófilo de montaña & 1,629 & 4 & 1 \\
\hline & Selva alta perennifolia & $11-746$ & 33 & 5 \\
\hline
\end{tabular}

$z a$, para Oaxaca y Puebla; sin embargo, en la publicación tampoco se hace referencia al ejemplar colectado por Thomas MacDougall y anotado como Oteiza macdougallii por Fay. Una consulta con los curadores de NY y TEX permitió corroborar que hasta la fecha no hay una formalización de este epíteto, a pesar de que existen los materiales anotados con este nombre en sus herbarios.

En cuanto a los aspectos de conservación, los 483 sitios de colecta pueden ser extrapolados en la relocalización de algunos taxones en particular, sobre todo en aquellos que se requiere evaluar o proteger para evitar el saqueo y comercio ilegal o para planear estrategias de conservación in y ex situ. De las especies que MacDougall colectó, 23 están enlistadas como especies en alguna categoría de riesgo (NOM-059SEMARNAT-2010; Cuadro 6). En la Lista Roja de la IUCN (IUCN, 2014) se encontró que solo cuatro de las 23 han sido evaluadas en los años de 2013 y 2014: Cypripedium irapeanum La Llave \& Lex.,VU B2ab(ii, iii, v), Epiphyllum chrysocardium Alexander, DD (como sinónimo de Selenicereus chrysocardium (Alexander) Kimnach), Magnolia schiedeana Schltdl., VU B1ab(iii), y Mammillaria deherdtiana Farwig, VU B1ab(iii, v), de acuerdo a las categorías de la IUCN (2012). También, es muy conocido que todas las especies de Cactáceas y Orquídeas se encuentran en los apéndices I y/o II de la Convención sobre el Comercio Inter- nacional de especies amenazadas de Fauna y Flora Silvestre (CITES, 2013). El objetivo de ubicar, georreferenciar y reconstruir el itinerario botánico de MacDougall, contribuyó en la localización de 483 sitios y su correspondencia con la vegetación primaria. MacDougall y colaboradores exploraron con mayor frecuencia el bosque mesófilo de montaña (BMM). En México, es un tipo de vegetación, que a pesar de ser muy fragmentado, es valioso porque alberga $25 \%$ de la biodiversidad del país. Asimismo, el BMM más extenso en el país se encuentra en el estado de Oaxaca, en la Sierra Madre Oriental y Sierra Norte de Oaxaca, donde la precipitación excede los 5,000 mm, particularmente a altitudes entre los 1,600 y 2,500 m (González-Espinosa et al., 2011). El estado tiene la mayor riqueza de especies vegetales a nivel nacional con 9,054 especies nativas (7.6\% de endémicas del estado; García-Mendoza y Meave, 2011).

\section{Conclusiones}

La base de datos electrónica de las colecciones de MacDougall ya se encuentra disponible y de libre acceso por medio de la REMIB de la CONABIO. Por otro lado, no se logró reconstruir el itinerario completo de colectas de MacDouga11, debido a la forma de cómo numeraba sus colectas, de tal manera que solo se pueden demarcar algunas rutas que él y 
Cuadro 6. Especies en la NOM-059-SEMARNAT-2010. A = amenazada, $\mathrm{P}=$ en peligro de extinción, $\mathrm{Pr}=$ sujeta a protección especial. Categorías que aplican a todo México.

\begin{tabular}{ll}
\hline Especie & Categoría NOM-059- \\
& SEMARNAT-2010 \\
\hline Agave guiengola & $\mathrm{A}$ (endémica) \\
Aporocactus flagelliformis & $\mathrm{P}$ (endémica) \\
Beschorneria albiflora & $\mathrm{Pr}$ \\
Chamaedorea elatior & $\mathrm{A}$ \\
Chamaedorea graminifolia & $\mathrm{A}$ \\
Chamaedorea tenella & $\mathrm{P}$ (endémica) \\
Chiranthodendron pentadactylon & $\mathrm{A}$ \\
Cypripedium irapeanum & $\mathrm{A}$ \\
Dahlia tenuicaulis & $\mathrm{Pr}$ \\
Dendrosida breedlovei & $\mathrm{A}$ \\
Epiphyllum chrysocardium & $\mathrm{A}$ (endémica) \\
Graptopetalum macdougallii & $\mathrm{P}$ (endémica) \\
Laelia anceps & $\mathrm{P}$ (endémica) \\
Laelia superbiens & $\mathrm{A}$ \\
Magnolia dealbata & $\mathrm{P}$ \\
Magnolia schiedeana & $\mathrm{A}$ \\
Mammillaria deherdtiana & $\mathrm{A}$ (endémica) \\
Monstera tuberculata & $\mathrm{A}$ \\
Oncidium leucochilum & $\mathrm{A}$ \\
Phymosia rosea & $\mathrm{Pr}$ \\
Reinhardtia elegans & $\mathrm{A}$ (endémica) \\
Synechanthus fibrosus & $\mathrm{P}$ \\
Tillandsia imperialis & $\mathrm{A}$ \\
\hline &
\end{tabular}

sus colaboradores siguieron. Se logró documentar los tipos de vegetación y las altitudes donde sus colecciones fueron realizadas. Por último, se logró enlistar algunas de las especies que se encuentran en alguna categoría de riesgo, tanto en la NOM-059-SEMARNAT-2010 como en la actual Lista Roja de la IUCN.

El documentar los sitios de colecta fue de gran importancia, ya que estos permiten: conocer la distribución de las especies, brindar información básica para análisis, tales como los de riqueza de la biodiversidad (pasado y actual), además de proyectar las necesidades de los escenarios futuros; asimismo, nos proporcionan elementos para llevar a cabo la evaluación de estados de conservación de las especies. Finalmente, son útiles para análisis espaciales y modelos de cobertura vegetal. MacDougall fue de los primeros exploradores que se aventuraron en la región de los Chimalapas. En 2013 se cumplieron 40 años de su fallecimiento, el contar ahora con una base electrónica de datos en la CONABIO, los nombres de sus colecciones ya es un legado disponible.

Hoy en día, la información en torno a la diversidad de un país y sus cambios a través del tiempo es fundamental para planear las estrategias de conservación. El presente trabajo apoya y cumple con el objetivo uno: documentar e integrar información relativa a la diversidad vegetal de la Estrategia Mexicana para la Conservación Vegetal 2012-2030 (CO-
NABIO, 2012). Los datos georreferenciados que posee la base dan los elementos para investigadores especializados, que tengan interés en evaluar a las especies o proponer medidas de protección para un conjunto de ellas, como serian áreas de reserva o colecta especifica de semillas/frutos para su conservación ex situ.

\section{Agradecimientos}

A los doctores Thomas Wendt y Nicholas Hind, por proporcionar datos inéditos de Oteiza macdougallii, la cual continúa como especie inédita. Wendt también ha proporcionado materiales de MacDougall depositados en el herbario TX. A los revisores de este trabajo que con sus comentarios y sugerencias que mejoraron la claridad de este artículo.

\section{Literatura citada}

APG III. Angiosperm Phylogeny Website. 2012. Version 12. $<$ http://www.mobot.org/MOBOT/research/APweb/> (consultado 30 abril 2015)

ASTER. Advanced Spaceborne Thermal Emission and Reflection Radiometer. 2009. Global Digital Elevation Modem. The Ministry of Economy, Trade and Industry of Japan (METI) and National Aeronautics and Space Administration (NASA). Versión 1. http://asterweb.jpl.nasa.gov/gdem.asp (consultado 30 abril 2015)

Bebber D.P., Carine M.A., Wood J.R.I., Wortley A.H., Harris D.J., Prance G.T., Davidse G., Paige J., Pennington T.D.M, Robson K.B. y Scotland R.W. 2010. Herbaria are a major frontier for species discovery in flowering plants. PNAS 107(51): 22169-22171. doi: 10.1073/pnas. 1011841108 (consultado 30 abril 2015 )

Borhidi A., Darók J., Kocsis M., Stranczinger S. y Kaposvári F. 2004. Critical revision of the Deppea complex (Rubiaceae, Hamelieae). Acta Botanica Hungarica 46:77-89.

Butterworth C.A. y Wallace R.S. 2004. Phylogenetic studies of Mammillaria (Cactaceae)-Insights from chloroplast sequence variation and hypothesis testing using the parametric bootstrap. American Journal of Botany 91:1086-1098.

CITES. Convención sobre el Comercio Internacional de especies amenazadas de Fauna y Flora Silvestre. 2013. http://www.cites. org/esp/news/sundry/2013/20131030_checklist.php (consultado 30 abril 2015)

CONABIO. Comisión Nacional para el Conocimiento y Uso de la Biodiversidad. 2003. México: Imagen desde el espacio. Mosaico 2002 de imágenes Modis sin nubes del satélite Terra, bandas 1,4,3 (RGB), resolución espacial 250 metros, sobre un modelo digital de terreno. Comisión Nacional para el Conocimiento y Uso de la Biodiversidad, México, D.F.

CONABIO. Comisión Nacional para el Conocimiento y Uso de la Biodiversidad. 2008. Georreferenciación de Localidades de Colecciones Biológicas: Manual de Procedimientos. Comisión Nacional para el Conocimiento y Uso de la Biodiversidad, México, D.F.

CONABIO. Comisión Nacional para el Conocimiento y Uso de la Biodiversidad. 2012. Estrategia Mexicana para la Conservación Vegetal, 2012-2030. Comisión Nacional para el Conocimiento y Uso de la Biodiversidad, México, D.F. 
Cronquist A. 1981. An Integrated System of Classification of Flowering Plants. Columbia University Press, Nueva York.

Fay J.J. 1978. Revision of Perymenium (Asteraceae-Heliantheae) in Mexico and Central America. Allertonia 1:235-296.

García-Mendoza A.J. y Meave J.A. Eds. 2011. Diversidad Florística de Oaxaca: de Musgos a Angiospermas (Colecciones y Lista de Especies). Universidad Nacional Autónoma de México, Comisión Nacional para el Conocimiento y Uso de la Biodiversidad, México, D.F.

Goodwin G.G. y MacDougall T.B. 1955a. Three new cotton rats from Tehuantepec, Mexico. American Museum Novitates 1705: $1-5$.

Goodwin G.G. y MacDougall T.B. 1955b. Two new white-footed mice from Oaxaca, Mexico. American Museum Novitates 1732:1-5.

Goodwin G.G. y MacDougall T.B. 1955c. New tree climbing rats from Mexico and Colombia. American Museum Novitates 1738: $1-5$.

Goodwin G.G. y MacDougall T.B. 1956a. A preliminary report on the mammals collected by Thomas MacDougall in Southeastern Oaxaca, Mexico. American Museum Novitates 1757:1-15.

Goodwin, G.G. y MacDougall T.B. 1956b. Seven new mammals from Mexico. American Museum Novitates 1791:1-10.

Goodwin G.G. y MacDougall T.B. 1958. Two new mammals from Mexico. American Museum Novitates 1871:1-3

Goodwin G.G. y MacDougall T.B. 1959a. A new pygmy mouse of the genus Baiomys from Oaxaca, Mexico. American Museum Novitates 1929:1-2.

Goodwin G.G. y MacDougall T.B. 1959b. Descriptions of some new mammals. American Museum Novitates 1967:1-8.

Goodwin G.G. y MacDougall T.B. 1963. A new species of Bobcat (Lynx rufus) from Oaxaca, Mexico. American Museum Novitates 2139:1-7.

Goodwin G.G. y MacDougall T.B. 1964. A species and a new subspecies of Peromyscus from Oaxaca, Mexico. American Museum Novitates 2183:1-8.

Goodwin G.G. y MacDougall T.B. 1966. A new species of vole (genus Microtus) from Oaxaca, Mexico. American Museum Novitates 2243:1-4.

Goodwin G.G. y MacDougall T.B. 1969. Mammals from the State of Oaxaca, Mexico, in the American Museum of Natural History. Bulletin of the American Museum of Natural History 141:1-269.

González-Espinosa M., Meave J.A., Lorea-Hernández F.G., Ibarra-Manríquez G. y Newton A.C. (Eds.). 2011. The Red List of Mexican Cloud Forest Trees. Fauna \& Flora International, Cambridge. https://portals.iucn.org/library/node/10146 (consultado 30 abril 2015)

INEGI. Instituto Nacional de Estadística, Geografía e Informática. 2003. Vegetación Primaria Escala 1:1000000. Instituto Nacional de Estadística, Geografía e Informática, Aguascalientes.

IUCN. International Union for Conservation of Nature. 2012.
IUCN Red List categories and criteria: version 3.1. $2^{\mathrm{a}}$ ed. Gland. https://portals.iucn.org/library/node/10315 (consultado 30 abril 2015 )

IUCN. International Union for Conservation of Nature. 2014. The IUCN Red List of Threatened Species. 2014.3 http://www.iucnredlist.org/ (consultado 30 abril 2015 )

Kirkbride Jr.J.H. 1984. Review of Omiltemia (Rubiaceae). Systematic Botany 9:410-414.

Lorence D.H. y Dwyer J.D. 1988. A revision of Deppea (Rubiaceae). Allertonia 4:389-436.

MacDougall T.B. 1971. The Chima wilderness. Explorers Journal 49:86-103.

SEMARNAT. Secretaría de Medio Ambiente y Recursos Naturales. NOM-059-SEMARNAT-2010. Norma Oficial Mexicana, Protección ambiental-Especies nativas de México de flora y fauna silvestres-Categorías de riesgo y especificaciones para su inclusión, exclusión o cambio-Lista de especies en riesgo.

Panero J.L., Villaseñor J.L. y Medina R. 1993. New species of Asteraceae-Heliantheae from Latin America. Contributions from the University of Michigan Herbarium 19:171-193.

Peterson D.A. y MacDougall T.B. 1974. Guiengola: a fortified site in the Isthmus of Tehuantepec. Publication in Anthropology 10. Vanderbilt University, Nashville.

Robinson H.E. 1979. Study of the genus Schistocarpha (Heliantheae: Asteraceae). Smithsonian Contributions to Botany 42:120.

Root N.J. 1975. The Thomas Baillie MacDougall Collection in The American Museum of Natural History Library. Curator: The Museum Journal 18:276-280.

Shufeldt R.W. 1872. Reports of Explorations and Surveys: To Ascertain the Practicability of a Ship-Canal Between the Atlantic and Pacific Oceans, by the Way of the Isthmus of Tehuantepec. Government Printing Office, Washington, D.C.

Stix J.S. 1973. Tom MacDougall (obituary). Cactus and Succulent Journal 45:55.

Stix J.S. 1974. Yo soy botánico. Cactus and Succulent Journal 46:117-119.

Stix J.S. 1975a. Thomas Baille MacDougall-Naturalist and collector. Curator: The Museum Journal 18:270-276.

Stix J.S. 1975b. Signed and sealed to nature. The botanical career of Thomas MacDougall. Garden Journal 25:47-53.

Taylor E.H. y MacDougall T.B. 1949. New or unusual Mexican amphibians. American Museum Novitates 1437:1-22.

Wieczorek J., Guo Q. y Hijmans R. 2004. The point-radius method for georeferencing locality descriptions and calculating associated uncertainty. International Journal of Geographic Information Science 18:745-767.

Williams J.J. 1852. The Isthmus of Tehuantepec: Being the result of a survey for a railroad to connect the Atlantic and pacific oceans, made by the scientific commission under the direction of Major J. G. Barnard. D. Appleton \& Co., Nueva York. 